\title{
Investigating the molecular basis for heterophylly in the aquatic plant Potamogeton octandrus (Potamogetonaceae) with comparative transcriptomics
}

\author{
Dingxuan He ${ }^{1}{ }^{\text {, Pin Guo }}{ }^{2}$, Paul F Gugger ${ }^{3}$, Youhao Guo ${ }^{1}{ }^{\text {, Xing Liu }}{ }^{\text {Corresp.. }}{ }^{1}$, Jinming Chen ${ }^{\text {Corresp. }}{ }^{4}$ \\ 1 Laboratory of Plant Systematics and Evolutionary Biology, College of life Sciences, Wuhan University, Wuhan, Hubei, China \\ 2 Department of Biological Sciences, Graduate School of Science, The University of Tokyo, Tokyo, Japan \\ 3 Appalachian Laboratory, University of Maryland Center for Environmental Science, Frostburg, Maryland, USA \\ 4 Key Laboratory of Aquatic Botany and Watershed Ecology, Wuhan Botanical Garden, Chinese Academy of Sciences, Wuhan, China \\ Corresponding Authors: Xing Liu, Jinming Chen \\ Email address: xingliu@whu.edu.cn, jmchen@wbgcas.cn
}

Many plant species exhibit different leaf morphologies within a single plant, or heterophylly. The molecular mechanisms regulating this phenomenon, however, have remained elusive. In this study, the transcriptomes of submerged and floating leaves of an aquatic heterophyllous plant, Potamogeton octandrus Poir, at different stages of development, were sequenced using high-throughput sequencing (RNA-Seq), in order to aid gene discovery and functional studies of genes involved in heterophylly. A total of 81,103 unigenes were identified in submerged and floating leaves and 6,822 differentially expressed genes (DEGs) were identified by comparing samples at differing time points of development. KEGG pathway enrichment analysis categorized these unigenes into 128 pathways. A total of 24,025 differentially expressed genes were involved in carbon metabolic pathways, biosynthesis of amino acids, ribosomal processes, and plantpathogen interactions. In particular, KEGG pathway enrichment analysis categorized a total of 70 DEGs into plant hormone signal transduction pathways. The high-throughput transcriptomic results presented here highlight the potential for understanding the molecular mechanisms underlying heterophylly, which is still poorly understood. Further, these data provide a framework to better understand heterophyllous leaf development in P. octandrus via targeted studies utilizing gene cloning and functional analyses. 


\section{Investigating the molecular basis for heterophylly in the aquatic}

\section{2 plant Potamogeton octandrus (Potamogetonaceae) with comparative}

\section{3 transcriptomics}

4 Dingxuan $\mathrm{He}^{1}$, Pin $\mathrm{Guo}^{2}$, Paul F. Gugger ${ }^{3}$, Youhao Guo ${ }^{1}$, Xing Liu ${ }^{1 *}$, Jinming Chen ${ }^{*}$

51 Laboratory of Plant Systematics and Evolutionary Biology, College of Life Science, Wuhan

6 University, Wuhan, China

72 Department of Biological Sciences, Graduate School of Science, The University of Tokyo,

8 Tokyo, Japan

93 Appalachian Laboratory, University of Maryland Center for Environmental Science, Frostburg,

10 Maryland, USA

114 Key Laboratory of Aquatic Botany and Watershed Ecology, Wuhan Botanical Garden, Chinese

12 Academy of Sciences, Wuhan, China

$13 *$ Corresponding authors:

14 Xing Liu:

15 Laboratory of Plant Systematics and Evolutionary Biology, College of Life Science, Wuhan

16 University, Wuhan 430072, China;

17 Tel.: +86-27-68756612; Fax: +86-27-68752560; E-mail: xingliu@whu.edu.cn

18 Jin-Ming Chen:

19 Key Laboratory of Aquatic Botany and Watershed Ecology, Wuhan Botanical Garden, Chinese

20 Academy of Sciences, Wuhan 430074, China; 
21 Tel: 86-27-87617211; Fax: 86-27-87510526; E-mail: jmchen@wbgcas.cn

\section{ABSTRACT}

Many plant species exhibit different leaf morphologies within a single plant, or heterophylly.

24 The molecular mechanisms regulating this phenomenon, however, have remained elusive. In this

25 study, the transcriptomes of submerged and floating leaves of an aquatic heterophyllous plant,

26 Potamogeton octandrus Poir, at different stages of development, were sequenced using high-

27 throughput sequencing (RNA-Seq), in order to aid gene discovery and functional studies of

28 genes involved in heterophylly. A total of 81,103 unigenes were identified in submerged and

29 floating leaves and 6,822 differentially expressed genes (DEGs) were identified by comparing

30 samples at differing time points of development. KEGG pathway enrichment analysis

31 categorized these unigenes into 128 pathways. A total of 24,025 differentially expressed genes

32 were involved in carbon metabolic pathways, biosynthesis of amino acids, ribosomal processes,

33 and plant-pathogen interactions. In particular, KEGG pathway enrichment analysis categorized a

34 total of 70 DEGs into plant hormone signal transduction pathways. The high-throughput

35 transcriptomic results presented here highlight the potential for understanding the molecular

36 mechanisms underlying heterophylly, which is still poorly understood. Further, these data

37 provide a framework to better understand heterophyllous leaf development in $P$. octandrus via

38 targeted studies utilizing gene cloning and functional analyses.

\section{INTRODUCTION}

40 The presence of two or more leaf forms on a single plant, or heterophylly, is widely

41 observed across diverse plant species, and most notably in aquatic plants (Minorsky, 2003; Zotz,

42 Wilhelm \& Becker, 2011). In some cases, heterophylly is believed to be an adaptive response to 
43 the environment, and it has been linked to an increase in fitness (Cook \& Johnson, 1968; Wells

44 \& Pigliucci, 2000; Minorsky, 2003). For example, the heterophylly of aquatic plants may

45 increase their fitness by decreasing leaf damage, decreasing water loss, enhancing photosynthesis,

46 or promoting sexual reproductive success (Winn, 1999a, b; Wells \& Pigliucci, 2000; Minorsky,

47 2003; Zhang et al., 2009; Zotz, Wilhelm \& Becker, 2011). Accordingly, heterophylly has been

48 used as a model system for studying gene-environment interactions (Pigliucci, 2010; Nakayama

49 et al., 2014).

50 In the past century, numerous studies have described morphological changes in

51 heterophyllous plants in response to environmental factors such as $\mathrm{CO}_{2}$ concentration, oxygen

52 capacity, salt concentration, temperature, water levels, seasonal changes, and light intensity and

53 quality (McCallum, 1902; Arber, 1920; Fassett, 1930; Sculthorpe, 1967; Cook \& Johnson, 1968;

54 Bodkin, Spence \& Weeks, 1980; Deschamp \& Cooke, 1984; Titus \& Sullivan, 2001). More

55 recent studies have also revealed that plant hormones, including ethylene, abscisic acid (ABA),

56 and gibberellin (GA) can affect heterophyllous leaf formation in many plant species, including

57 Potamogeton nodosus (Anderson, 1978), Hippuris vulgaris (Kane \& Albert, 1987), Marsilea

58 quadrifolia (Liu, 1984), Callitriche heterophylla (Deschamp \& Cooke, 1985), Ranunculus

59 flaellaris (Young \& Horton, 1985; Young, Dengler \& Horton, 1987), Ludwigia arcuata

60 (Kuwabara, 2003), and Rorippa aquatica (Nakayama et al., 2014). For example, ethylene gas has

61 been shown to induce the development of submerged-type leaves on terrestrial shoots of $L$.

62 arcuate. $\mathrm{ABA}$, however, induces the formation of terrestrial-type leaves on submerged shoots

63 (Kuwabara, 2003). However, the molecular mechanisms regulating the alterations of leaf forms

64 in heterophyllous plant species remain largely unclear.

65 Plant leaves arise from a group of initial cells termed shoot apical meristems (SAMs). The 
66 molecular mechanisms responsible for the initiation and maintenance of SAMs and polar

67 processes of leaf expansion have been recently studied in detail (Hay \& Tsiantis, 2006; Tsukaya,

68 2006; Uchida et al., 2007, 2010; Shani et al., 2010; Moon \& Hake, 2011). For example, SAMs

69 are characterized by expression of the Class I KNOTTED1-LIKE HOMEOBOS (KNOX) gene

70 (Smith et al., 1992; Jackson, Veit \& Hake, 1994), and down-regulation of the KNOX gene in

71 regions where the leaf primordia will initiate is one of the earliest indications of leaf

72 development (Moon \& Hake, 2011). However, few studies have investigated the molecular

73 biological changes that occur during the course of heterophyllous transitions. Hsu et al. (2001)

74 identified several early-response ABA-regulated genes, designated $A B R H$ (ABA-responsive

75 heterophylly), in the aquatic fern M. quadrifolia. ABRH genes encode transcription factors,

76 protein kinases, membrane transporters, metabolic enzymes, and structural proteins. In addition,

77 Chen et al. (2011) demonstrated that 9-cis-epoxycarotenoid dioxygenase 3 (NCED3) plays a key

78 role in regulating $\mathrm{ABA}-$ mediated heterophylly via endogenous $\mathrm{ABA}$ in two different lily

79 varieties. Nakayama et al. (2014) also showed that regulation of GA levels via KNOX1 genes is

80 primarily responsible for modulating heterophylly in R. aquatica. Heterophylly occurs across

81 diverse taxa and may have evolved through convergent evolution (Minorsky, 2003), and thus

82 different developmental processes and molecular mechanisms may exist among different species.

83 Further, heterophylly within a single plant is controlled through multiple signalling pathways

84 (Lin \& Yang, 1999; Hsu et al., 2001). Thus, additional study systems in diverse heterophyllous

85 plants and the use of large comparative datasets generated at the whole genomic or

86 transcriptomic levels would aid in elucidating the complex molecular mechanisms that regulate

87 heterophylly. 
Here, we investigated the molecular mechanisms underlying heterophylly in Potamogeton

89 (Potamogetonaceae), which is a genus that comprises more than eight heterophyllous species

90 (Guo et al., 2010). P. octandrus Poir $(2 \mathrm{n}=28)$, a heterophyllous pondweed with submerged and

91 floating leaf forms, is a perennial aquatic herb that is self-compatible and can reproduce

92 vegetatively through rhizomes, or sexually by selfing and outcrossing seeds. $P$. octandrus

93 produces many floating leaves that are flat and ovate with sharp leaf tips and submerged leaves

94 that are linear in shape (Fig. 1). During the initial development stage, all seedlings are submerged

95 under water and the stem apex can produce sessile, linear, and entire submerged leaves arranged

96 in a decussate phyllotaxy (Fig. 1a). When submerged stems reach the surface of the water, the

97 stems begin plagiotropic growth, and the stem apex can produce both floating and submerged

98 leaves as the stem elongates (Fig. 1b).

99 The transcriptomes of submerged and floating $P$. octandrus leaves at different time points

100 along the developmental trajectory were sequenced using the Illumina RNA-Seq platform. De

101 novo assembly of the DNA reads generated from submerged and floating leaf materials were

102 then used as the reference transcriptome, and short sequence reads that were generated at several

103 time points within the development of submerged and floating leaves were mapped to the

104 assembled transcriptome in order to identify genes exhibiting differential expression between

105 leaf morphologies. These results provide a crucial reference transcriptome for investigating the

106 regulatory mechanisms underlying each leaf form, in addition to a list of candidate genes that are

107 likely to be involved in the transition to heterophylly. Further, these results add to the growing

108 body of data aimed at understanding the regulatory mechanisms of heterophylly among

109 heterophyllous plants. 


\section{MATERIALS AND METHODS}

\section{Plant materials}

112 A P. octandrus plant was collected from a Tongcheng population $\left(29^{\circ} 16^{\prime} 05.6^{\prime \prime} \mathrm{N}\right.$,

$113113^{\circ} 48^{\prime} 46.9^{\prime \prime}$ E) in the Hubei Province, China, and used for transcriptomic analysis. The plant

114 was transplanted to a greenhouse at Wuhan University in April 2015 and the seeds were

115 harvested in August 2015. Seedlings were cultured in a pool at Wuhan University and floating

116 and submerged leaf forms were sampled at the following time points: (1) floating leaves with

117 length less than $0.5 \mathrm{~cm}$ (JFL; 72 hours); (2) floating leaves with length more than $1 \mathrm{~cm}$ (AFL; 84

118 hours); (3) submerged leaves with length less than $1.5 \mathrm{~cm}$ (JSL; 96 hours); (4) submerged leaves

119 with length more than $3 \mathrm{~cm}$ (ASL; 120 hours), in addition to (5) leaf-shoots of plants with stems

120 that have an apex just reaching the water surface (shoot; 24 hours). In the shoot category,

121 identification of which leaf form the shoot will develop into is not yet possible . The "shoot" was

122 considered as the initial stage of either floating or submerged leaves. Fifteen samples with three

123 biological replicates for each leaf/shoot form were collected in total. Sampled tissues were

124 immediately frozen in liquid nitrogen and stored at $-80^{\circ} \mathrm{C}$ until further analysis.

\section{RNA extraction, cDNA library construction and sequencing}

126 Total RNA from each sample was extracted using the TRIzol reagent (Invitrogen, Carlsbad,

127 CA, USA) according to the manufacturer's instructions. RNA was then treated with RNase-free

128 DNase I (Fermentas, ThermoFisher, Waltham, MA, USA). The quantity and quality of RNA was

129 assessed using 1\% agarose gel electrophoresis of extracted RNA, in addition to analyses with an

130 RNA 6000 Nano Assay Kit, and an Agilent 2100 Bioanalyzer (Agilent Technologies, Palo Alto,

131 CA, USA). Total extracted RNA was stored at $-80^{\circ} \mathrm{C}$. 
133 synthesized using a SuperScript VILO cDNA Synthesis Kit (Invitrogen., Carlsbad, CA, USA)

134 according to the manufacturer's protocol. A total of $10 \mu \mathrm{g}$ of RNA was purified using oligo (dT)

135 magnetic beads in order to enrich for poly (A) mRNA. Fragmentation buffer was then added to

136 split mRNA into short fragments. Fragments were used as templates to synthesize first-strand

137 cDNA using random hexamer-primers (Invitrogen, Carlsbad, CA, USA) and $1000 \mathrm{U}$ of

138 SuperScriptTM II reverse transcriptase (Invitrogen, Carlsbad, CA, USA). RNase H and DNA

139 polymerase I were used to synthesize second-strand cDNA. The short fragments were then

140 amended with adapters and end repair ligation. The products were first purified using a

141 QIAquick ${ }^{\circledR}$ Polymerase Chain Reaction (PCR) Extraction Kit (Qiagen, Hilden, Germany), and

142 then enriched with PCR (15 cycles) to create the final cDNA libraries. The 15 cDNA libraries

143 were then sequenced to obtain 150 bp paired-end reads using the Illumina HiSeq ${ }^{\mathrm{TM}} 2500$

144 sequencing platform (Illumina Inc., San Diego, CA, USA). The raw sequencing data were

145 deposited into the NCBI Short Reads Archive (SRA) under the accession numbers SRR6649465-

146 67, SRR6649858-60, SRR6649878-80, SRR6650048-50, SRR6655609-11.

\section{Sequence assembly and gene annotation}

148 The raw reads were cleaned by removing low quality reads, adapter reads, and reads with

$1495 \%$ or more unknown nucleotides. Transcriptome de novo assembly was performed with the

150 clean reads that resulted from all 15 samples using the Trinity program (Grabherr et al., 2011)

151 with the min_kmer_cov parameter set to 2 by default. Overlapping sequence reads were first

152 combined to form contigs without gaps, and reads were then reverse mapped to the contigs.

153 Paired-end sequencing allowed the inference of contigs arising from the same transcript, and

154 distances between these contigs were determined. Contigs were connected in Trinity and 
155 sequences that could not be extended on either end were identified, followed by construction of

156 'unigenes' that represented transcripts from the same locus. The completeness of the $P$.

157 octandrus transcriptome assembly was estimated using reciprocal BLAST searches against

158 annotated proteins from the genome of a seagrass species closely related to P. octandrus, Zostera

159 marina (Olsen et al., 2016) and an E-value threshold of 1e-5.

160 The putative functions of unigenes were then assessed using BLASTx (E-value $\left.\leq 10^{-5}\right)$ and 161 several protein databases including the: NCBI non-redundant protein (nr), Swiss-Prot, Clusters

162 of Orthologous Groups (COG), euKaryotic Orthologous Groups (KOG), eggNOG, Protein

163 family (Pfam), Gene Ontology (GO), and Kyoto Encyclopedia of Genes and Genomes (KEGG)

164 databases. The sequencing directions of unigenes were determined based on the best alignments.

165 The ESTScan program (version 3.0.1, http://www.ch.embnet.org/software/ESTScan.html) was

166 used to determine sequencing direction when a unigene could not be aligned with any of the

167 above databases. Blast2GO 2.5.0 (Conesa et al., 2005) was used to compare and determine

168 unigene Gene Ontology (GO) annotations. GO functional classifications for all annotated genes

169 were obtained with the program WEGO (Ye et al., 2006), which was also used to plot the

170 distribution of gene functions.

\section{Analysis of differentially expressed genes (DEGs)}

172 To quantify the abundance of transcripts, clean reads from each sample were then mapped

173 onto the assembled reference transcriptome using the Bowtie2 read-mapping, alignment software

174 (Langmead \& Salzberg, 2012). Gene expression levels and comparisons of gene fragments per

$175 \mathrm{~kb}$ per million (FPKM) fragments values (Marioni et al., 2008) were then assessed using

176 likelihood ratio tests in order to statistically identify differentially expressed genes (DEGs). A

177 previously developed algorithm (Audic and Claverie, 1997) was used to assess DEGs between 
178 samples from different leaf form stages (control/experimental: shoot/JFL, shoot/AFL, JFL/AFL,

179 shoot/JSL, shoot/ASL, JSL/ASL). False discovery rate (FDR) control was used to correct for $P$

180 values for multiple hypotheses. Genes with changes in expression that were two-fold or greater

181 and had FDR values $\leq 0.01$ were identified as differentially expressed and then absolute values

182 of $\log _{2}$ (foldchange) $\geq 1$ were regarded as DEGs.

183 GO functional analysis was used to assess GO functional enrichment and GO functional 184 annotation for the DEGs. All DEGs were mapped to each term of the GO database. We then 185 calculated the gene abundances for each GO term. A hypergeometric test was then applied to 186 identify significantly enriched GO terms in DEGs relative to the genome background. $P$-values

187 from the GO enrichment analysis were adjusted using Bonferroni's correction. A corrected $P_{-}$

188 value $\leq 0.05$ was selected as the threshold for significantly enriched GO terms. DEGs were then

189 mapped to terms in the KEGG database to obtain enriched pathway terms (Kanehisa \& Goto,

190 2000). Pathways with an FDR value of $\leq 0.05$ were considered significant.

\section{Quantitative real-time PCR (qPCR) analysis}

192 To validate the transcriptomic data, 16 DEGs were randomly selected and their expression

193 profiles were investigated by qPCR. Total RNA was extracted from P. octandrus leaves in the

194 five groups and cDNA libraries were constructed as described above. A SYBR Premix Ex Taq ${ }^{\mathrm{TM}}$

195 Kit (TaKaRa) was used for the qPCR reactions in a BioRad Real-Time thermal cycler system

196 (BioRad, Hercules, CA, USA). Gene-specific primers were designed using Primer Premier5, and

197 the primer sequences are provided in File S5. The PCR cycling regimen was developed

198 according to the manufacturer's protocol and consisted of $95^{\circ} \mathrm{C}$ for $30 \mathrm{~s}, 40$ cycles of $95^{\circ} \mathrm{C}$ for 5

$199 \mathrm{~s}$ and $58^{\circ} \mathrm{C}$ for $30 \mathrm{~s}$. Each reaction was performed in triplicate and $\beta$-actin was used as a 
200 reference gene. The $2^{-\Delta \Delta \mathrm{Ct}}$ method was used to calculate relative gene expression levels (Livak \& 201 Schmittgen, 2001).

\section{RESULTS}

203 Illumina sequencing and de novo assembly

204 After removal of duplicate sequences, adaptor sequences, low quality reads, and reads with 205 ambiguous bases, a total of $135.83 \mathrm{Gbp}$ clean sequence data with Q30 $\geq 96.0 \%$ were obtained 206 from the 15 cDNA libraries. Clean reads were pooled and de novo assembled into 81,103

207 unigenes, which had an average length of $841 \mathrm{bp}(\mathrm{N} 50=1713 \mathrm{bp})$ (Table 1). The most abundant 208 length fraction of Unigenes were $201-300 \mathrm{bp}$, followed by $500-1,000 \mathrm{bp}$ and $300-500 \mathrm{bp}$ 209 sequences (Table 1). Cleaned reads were then mapped to the unigenes to assess sequencing 210 randomness, which was found to be sufficient (Additional file 1). Further read mapping statistics

211 for each sample are provided in Additional file 2. About 91.1\% $(18,738 / 20,559)$ of all annotated

212 proteins from a closely related seagrass species could be identified in the P. octandrus

213 transcriptome, indicating that our transcriptome assembly likely covers the majority of the core

$214 P$. octandrus transcriptome. In contrast, only $60.5 \%(101,218 / 167,270)$ of $P$. octandrus

215 transcripts could be annotated using the closely related seagrass species proteins, suggesting that

216 the remainder of the unannotated transcripts are from lineage-specific genes or long non-coding 217 RNAs.

\section{Functional annotation and classification of unigenes}

219 A total of 48,235 (56\% of all unigenes) consensus sequences were annotated using BLASTx $220\left(E\right.$-value $\left.\leq 1 \times 10^{-5}\right)$ against several public protein databases including NCBI-nr, Swiss-Prot, 221 COG, KOG, eggNOG, Pfam, GO, and KEGG (Additional file 3). Among the annotated unigenes, 
22224,025 were categorized into 52 functional subcategories under three main GO categories:

223 cellular components, molecular functions, and biological processes (Fig. 2). The GO category

224 "biological processes" represented the largest number of GO annotations, with metabolic

225 processes, cellular processes, and single-organism processes comprising the three most abundant

226 top-level subcategories. In the cellular components category, the "cell" and "cell part" categories

227 were most represented, while in the molecular functions category, the "catalytic activity"

228 subgroup was prominent, followed by the "binding" group. In the COG and KOG functional

229 classifications, "translation, ribosomal structure and biogenesis" associated proteins represented

230 the largest group (Additional files 4 and 5). Lastly, a total of 22,346 unigenes were assigned to

231128 KEGG pathways.

232 DEG identification and functional analysis

233 A total of 6,822 differentially expressed genes (DEGs) were identified by comparing

234 samples from each leaf form group (control/experiment: shoot/JFL, shoot/AFL, JFL/AFL,

235 shoot/JSL, shoot/ASL, JSL/ASL; Additional file 6). A Venn diagram was used to visualize the

236 numbers of overlapping and unique DEGs among and within groups (Fig. 3a, b). The shoot/JFL

237 comparison contained the most DEGs $(3,706)$, whereas the smallest number of DEGs $(64)$ was

238 detected in the JSL/ASL comparison. Among the DEGs associated with the development of

239 floating leaves, 2,186, 1,621 and 78 were up-regulated, while 1,835, 1,679 and 332 were down-

240 regulated in comparisons of shoot/JFL, shoot/AFL, and JFL/AFL, respectively. During the

241 development of submerged leaves, 2,413, 2,242 and 45 DEGs were found to be up-regulated,

242 while 1,458, 1,284 and 33 were down-regulated in the shoot/JSL, shoot/ASL, and JSL/ASL

243 comparisons, respectively (Fig. 3c). These results indicated that the greatest differential

244 expression occurred in the early stages of development for both floating and submerged leaves 
245 (shoot/JFL and shoot/JSL). Further, the number of up-regulated DEGs was higher than the

246 number of down-regulated DEGs during the development of submerged leaves. However, the

247 number of up-regulated and down-regulated DEGs was essentially equivalent during the

248 development of floating leaves.

249 The number of DEGs that were annotated in the shoot/JSL and shoot/ASL comparisons

250 during floating leaf development was greater than those in the JSL/ASL comparison (Fig. 4). A

251 similar pattern was found in comparisons for the development of submerged leaves (Fig. 5). The

252 highest number of DEGs for each comparison was identified in the "biological process"

253 category, while the most abundant sub-group terms were in the "cellular process", "metabolic

254 process" and "single-organism" categories for the different groups of submerged and floating

255 leaves. Enriched GO terms during the earlier time points of leaf development (JFL and JSL)

256 included "translation", "regulation of transcription, DNA-templated", "RNA methylation", and

257 "photosynthesis". However, the GO terms enriched during the later developmental time points

258 were assigned to "response to salt stress" (AFL and ASL), "salicylic acid biosynthetic process"

259 (AFL), and "negative regulation of programmed cell death" (ASL). In the cellular component

260 category, "cell" and "cell part" were the two most highly represented components throughout the

261 developmental time points for all leaves. The GO terms "ribosome" and "cytosolic small

262 ribosomal subunit" were enriched in different stages of leaf development, while the GO terms

263 "cytoplasmic membrane-bounded vesicle" (JFL) and "chloroplast envelope" (JSL) were enriched

264 in the earlier developmental time points. In the molecular function category, DEGs that mapped

265 to "catalytic activity" and "binding" were present in high proportions through all developmental

266 time points. The results of GO enrichment analysis are presented in Additional file 7.

267 Annotated sequences were then searched against the KEGG pathways database. Among the 
268 annotated DEGs, 1,490, 1,141, 135, 1,425, 1,362, and 30 were assigned to $114,114,57,114,111$,

269 and seven pathways in the shoot/JFL, shoot/AFL, JFL/AFL, shoot/JSL, shoot/ASL, and

270 JSL/ASL comparisons, respectively (Additional file 8). The pathways with the largest

271 proportions of DEGs were "ribosome", "biosynthesis of amino acids", "carbon metabolism", and

272 "plant-pathogen interaction" in the shoot/JFL, shoot/AFL, shoot/JSL, and shoot/ASL

273 comparisons, respectively (Additional file 8). However, these four pathways accounted for a

274 lower percentage of the proportion in the JFL/AFL and JSL/ASL groups, which may be

275 important in the early stages of development of floating and submerged leaves. "Plant hormone

276 signal transduction" was the most enriched cluster among the DEGs, indicating that proteins in

277 this category may be important in the morphological and physiological differentiation that occurs

278 in the early stages of leaf development.

279

280

281

282

283

284

285

286

287

288

289

290

\section{Analysis of transcription factors associated with heterophyllous leaf types}

To assess the function of transcription factors (TFs) during the development of heterophyllous leaves, we identified $P$. octandrus TF genes using the Plant Transcription Factor Database (http://planttfdb.cbi.pku.edu.cn/). In total, we identified 1,681 putative TF genes that were categorized into 48 families. Four-hundred and sixty-nine of the 1,681 putative TF genes that were from 42 families showed significant differential expression between the leaf groups (Fig. 6 and Additional file 9). Most of these genes (53\%) were expressed at the highest levels in the AFL, ASL, JFL, and JSL groups (Group 2; G2). Only 37\% were expressed at the highest levels in the "shoot" group (Group 1; G1). Lastly, an additional 10\% exhibited highest expression in the JFL group (Group 3; G3). We further investigated differential expression at the family-specific level (Fig. 7). The WRKY, C3H, and AP2 families of TFs, which play roles in various processes including stress response, leaf senescence, and leaf epidermal cell identity, 
291 were highly expressed in G2 (Additional file 10). Many TFs that mediate hormone signaling,

292 including ethylene signaling (ERF family) and auxin signaling (NAC family), were highly

293 expressed during the development of floating and submerged leaves (G2). The HD-Zip family

294 members that have been proposed as regulators of vascular development, stomatal complex

295 morphogenesis, leaf polarity, and epidermal cell differentiation, were most highly expressed in

296 G2. MYB, ARF, and B3 TFs are involved in light and hormone signaling pathways. These genes

297 were most expressed in the "shoot" (G1) group, where cell differentiation and cellular

298 morphogenesis are modulated. Homeobox genes, which participate in a number of

299 developmental events, were also highly expressed in G1. Several FAR1 and bHLH TFs, which

300 have been reported to function in light signaling and stomatal development, were enriched in the

301 earlier time point floating leaves (G3). These results suggest that transcriptional regulatory genes

302 are critical for a wide variety of developmental processes in the leaf transcriptome.

303 Hormone metabolism and signalling pathways among heterophyllous leaf samples

304 KEGG annotation revealed that most DEGs were classified in the "plant hormone signal

305 transduction" category. A total of 70 DEGs were annotated as diverse hormone-related genes,

306 including those involved in signalling with abscisic acid (ABA), auxin, cytokinin (CTK),

307 ethylene, jasmonic acid (JA), gibberellin (GA), brassinosteroid, and salicylic acid (SA).

308 Among hormone signaling genes, most were involved in auxin response pathways during

309 the development of both floating and submerged leaves. Genes encoding the auxin response

310 factor (ARF) were down-regulated, while genes encoding SAUR family members were

311 differentially expressed, with two DEGs up-regulated and two down-regulated. Genes encoding

312 an auxin influx transport protein (AUX1) and an auxin-responsive protein AUX/IAA were up-

313 regulated in floating leaves, whereas those genes were down-regulated in submerged leaves. 
314 Eight genes that were associated with ABA and GA pathways were highly expressed in

315 floating leaves, including those encoding PYR (pyrabactin resistance)/PYL (PYR1-like), SNF1

316 related protein kinase 2 (SnRK2), ABA responsive element binding factor (ABF), and

317 phytochrome-interacting factor (PIF). Genes that were related to GA and ethylene pathways

318 were also highly expressed in submerged leaves, including one that encoded gibberellin receptor

319 (GID1) and two encoding the ethylene-responsive transcription factor 1 (ERF1).

320 Among the 31 hormone-related genes that were up-regulated during the differing

321 developmental time points of floating leaves, four were involved in the ABA signal transduction

322 pathway, four were associated with the GA signal transduction pathway, five were responsive to

323 auxin stimulation, three were associated with ethylene-mediated signaling, two were responsive

324 to CTK stimulus, and three were related to brassinosteroid signaling. Most of these genes were

325 significantly up-regulated in the earlier time point of floating leaves. Additionally, 34 genes

326 involved in the hormone signal transduction pathway were up-regulated during the different

327 developmental points of submerged leaves. When compared with floating leaves, more genes

328 were involved in GA, ethylene and CTK signal transduction pathways during the development of

329 submerged leaves (Additional file 11).

330 Expression patterns of ABA- and GA-biosynthesis genes

331 In the ABA biosynthesis pathway, seven DEGs that were annotated to encode zeaxanthin 332 epoxidase (ZEP), 9-cis-epoxycarotenoid dioxygenase (NCED), and abscisic-aldehyde oxidase 333 (AAO), exhibited different levels of expression. Genes encoding ZEP and NCED were highly 334 expressed during the different stages of submerged leaves and particularly highly expressed in 335 floating leaves. Further, the expression of genes encoding AAO was down-regulated in the later 336 stages of development of submerged leaves. 
In the GA biosynthesis pathway, the expression of one DEG encoding gibberellin 20

338 oxidase (GA20ox), which is a key enzyme in GA biosynthesis, was down-regulated throughout

339 developmental time points, except during the later time points of floating leaves. Additionally,

340 two genes that encode gibberellin 2beta-dioxygenase (GA2ox), which has an inactive effect on

341 GAs, were down-regulated. These results suggested that GA signaling pathways are important in

342 early stages of leaf development.

343 Expression patterns of genes mapped to "stomatal complex morphogenesis" and "cuticle

344 development"

345 Of the genes that were functionally annotated, some were associated with the functional

346 groups “stomatal complex morphogenesis" and "cuticle development" throughout leaf

347 development time points. Twenty-three DEGs were associated with "stomatal complex

348 morphogenesis", including 12 that were up-regulated during different time points of floating leaf

349 development, and particularly highly expressed in the earlier time point for floating leaves. Three

350 DEGs were also down-regulated in submerged leaves. The up-regulated DEGs were annotated as

351 phosphoribulokinase, chloroplastic, auxin-binding protein ABP19a, leaf isozyme, and

352 phototropins.

353 A total of 11 DEGs were mapped to "cuticle development", four of which were up-

354 regulated during the different time points of floating leaves and three that were down-regulated.

355 The up-regulated DEGs include orthologs of 3-ketoacyl-CoA synthase 10 and beta-ketoacyl-

356 CoA synthase like protein. The large number of up-regulated stomatal morphogensis and cuticle

357 development genes may indicate regulation of leaf morphology and structure.

358 Validation of DEGs expression by qPCR

359 Sixteen candidate DEGs were selected for qPCR validation of expression levels. These 
360 genes were categorized into groups that were related to transcription factors, biological processes,

361 and hormone signaling. Five transcription factors were tested, including $N F-Y B, M Y B$, GRF,

362 NAC, and NF-YA (c260025.graph_c1, c265373.graph_c0, c267010.graph_c0, c268319.graph_c0,

363 c269000.graph_c0). Additionally, six genes that were involved in biological processes were

364 analyzed, including guard cell differentiation (c260078.graph_c1), stomatal complex

365 morphogenesis (c260159.graph_c0, c262330.graph_c0, c267324.graph_c0), wax biosynthetic

366 processes (c271037.graph_c1), and cuticle development (c271065.graph_c0). In hormone

367 signaling, $A U X / I A A$ (c264173.graph_c0), $A U X 1$ (c264621.graph_c1), $A R F$ (c268917.graph_c0),

368 GA2ox (c266852.graph_c0), and GA20ox (c268628.graph_c0) were selected. The gene

369 annotations of these candidate DEGs are provided in Additional file 12. The correlation between

370 RNA-Seq results (fold change) and qPCR results $\left(2^{-\Delta \Delta C T}\right.$ ) was measured by plotting the $\log _{2}$ fold

371 changes (Additional file 13a). The results suggested that the qPCR abundances of genes were

372 significant similar $\left(\mathrm{r}^{2}=0.65, P<0.01\right)$ to the RNA-Seq data (Additional file $\left.13 \mathrm{~b}\right)$.

\section{DISCUSSION}

374 A growing body of research has indicated that heterophylly is a common, adaptive response

375 by plants to increase their fitness through responses to environmental changes. However, the

376 molecular mechanisms underlying these processes have remained unclear. Comparative

377 transcriptomic analysis is an efficient method for discovering genes and investigating

378 biochemical pathways that are involved in physiological processes, and has recently become an

379 increasingly tractable methodology (Varshney et al., 2009; Ozsolak \& Milos, 2011; Shi et al.,

380 2011; Mutasa-Goettgen et al., 2012; Yang et al., 2014). Here, leaves of the aquatic

381 heterophyllous plant $P$. octandrus that has two leaf forms (submerged and floating), were

382 subjected to transcriptomic sequencing to identify differentially expressed genes among the two 
383 leaf morphologies at differing time points of leaf development. Overall, 81,103 unigenes were

384 assembled and 48,235 unigenes were annotated against public protein databases. A total of 6,822

385 differentially expressed genes (DEGs) were identified in comparisons between time points.

386 KEGG pathway enrichment analysis was used to sort a number of DEGs into plant hormone

387 signal transduction pathways, including Cytokinin, Auxin, Abscisic Acid, Gibberellin,

388 Brassinosteroid, Ethylene, and Jasmonic Acid related pathways.

389 The initial stage of leaf shoot development is enriched in metabolic processes, cellular

390 processes, and responses to stimuli. The expression levels of genes related to leaf

391 morphogenesis, photomorphogenesis, and hormone signaling increased during developmental

392 time points of submerged and floating leaves. This was especially evident in the younger floating

393 leaves, where genes involved in establishing stomata and leaf petioles were more highly

394 expressed than in other tissues. In older submerged leaves, genes associated with leaf senescence

395 were more highly expressed than in other tissues. These gene transcriptional regulations were

396 coincident with the developmental dynamics of heterophyllous leaves.

397 When grouped into KEGG pathways, most DEGs were associated with hormone signaling.

398 Exogenous and endogenous ABA and GA can regulate leaf form alteration in heterophyllous

399 plant species (Allsopp, 1962; Deschamp \& Cooke, 1984; Gee \& Anderson, 1996; Kuwabara,

400 2003). The ABA-mediated regulation of heterophyllous morphological changes has been studied

401 intensively (Wanke, 2011; Nakayama et al., 2012). Here, a large number of hormone-related

402 DEGs were associated with ABA and GA signal transduction pathways, suggesting that genes

403 responsive to ABA and GA might play an important role in heterophyllous leaf formation in $P$.

404 octandrus. The genes encoding the PYR/PYL ABA receptor, which interacts with PP2C

405 phosphatases and is a component of the ABA signaling pathway, were up-regulated in floating 
406 leaves. Further, genes encoding SnRF2 proteins were also up-regulated. Activated SnRF2

407 proteins can phosphorylate downstream targets, such as AREB/ABF transcription factors and

408 contribute to those signaling pathways (Cutler et al., 2010). Endogenous levels of ABA have

409 been shown to increase in leaves of water-stressed terrestrial plants and ABA plays a pivotal role

410 in drought stress in terrestrial plant species (Walton \& Li, 2013). Thus, ABA may have a similar

411 function in the initiation of heterophyllous leaves in response to the transition from submerged to

412 aerial conditions in P. octandrus (Goliber \& Feldman, 1989). Plant cells respond to

413 environmental stimuli through a series of intracellular signals. To minimize transpirational water

414 loss, ABA controls stomatal closure (Hirayama \& Shinozaki, 2010). This change is induced by

415 transcriptional reprogramming via the ABA signalosome complex (PYP/PYL-PP2C-SnRK2).

416 With increasing concentrations of ABA, the ABA signaling complex (PYP/PYL-PP2C-SnRK2)

417 can cause stomatal closure in guard cells in a calcium-independent manner and through key

418 biochemical messengers (Geiger et al., 2011).

419 Many enzymes in ABA biosynthesis are also induced during the drought stress response,

420 including the key enzymes ZEP, NCED, and AAO (Iuchi et al., 2001; Chen et al., 2011; Wanke,

421 2011). Our results indicated that 19 genes encoding ZEP, NCED, and AAO exhibited different

422 expression levels among leaf morphological groups. Six of the 19 genes were highly expressed

423 in floating leaves and up-regulated when compared to leaf shoots and submerged leaves, while

424 only one gene was down-regulated. These results suggest that differential expression of genes

425 that encode key enzymes in ABA biosynthesis might control ABA function at specific stages

426 during the initiation of heterophyllous leaf changes. Thus, ABA may play a complex role in

427 signalling transduction during heterophylly development in P. octandrus. 
Recently, ethylene has been suggested to influence the formation of heterophyllous leaves

429

430

431

432

433

434

435

436

437

438

439

440

441

442

443

444

445

446

447

in L. arcuata in a manner that is opposite of ABA, and also has been suggested to be an endogenous factor that induces the formation of submerged leaves (Kuwabara, Tsukaya \&

Nagata, 2001). Our results indicated that the expression of three genes encoding 1-

aminocyclopropane-1-carboxylate synthase (ACS), a rate-limiting enzyme in ethylene

biosynthesis, were highly expressed in floating leaves relative to leaf shoots and submerged leaves. GA likely has an antagonistic effect on heterophylly in aquatic plants and is also involved in the formation of submerged leaves. However, it induces heterophylly only indirectly through ethylene (Kuwabara, Tsukaya \& Nagata, 2001). Therefore, changes in endogenous ABA concentrations can influence the formation of aerial leaves and feedback antagonistically on ethylene and GA (Wanke, 2011). Our results also indicated that two genes encoding GA2ox, which has an inactive effect on GA, were down-regulated in submerged leaves. Taken together, these findings suggest that ABA signalling was enhanced in the aerial leaves of heterophyllous plant species.

Transcription factors (TFs) that target promoter regions regulate the concentrations of proteins as limiting factors. They also play an important role in responses to environmental stress (Yuan \& Perry, 2011) and plant development. Previous studies have demonstrated that many genes in the AP2/EREBP family participate in the transcriptional regulation of processes related to growth and development. For example, one member of the ERF gene family, ESR1, regulates shoot regeneration (Banno et al., 2001), while overexpression of $S H N$ gene that are AP2/EREBP transcription factors, increases cuticular wax (Aharoni et al., 2004). Further, the Glossyl5 gene from maize regulates leaf epidermal cell identity (Moose \& Sisco, 1996), and the LEAFY PETIOLE (LEP) gene influences leaf petiole development in Arabidopsis thaliana (van der 
451 Graaff et al., 2000). Here, we found that 48 TFs are predominantly expressed in P. octandrus and

45242 TFs are up- or down-regulated among morphological comparisons. Among these TFs, several

453 ERF homologs to SHN and LEAFY PETIOLE exhibited up-regulation at different time points of

454 leaf development, which is consistent with thicker waxy cuticles and extended leaf petioles on

455 floating leaves of $P$. octandrus. In addition, we detected a close homolog of maize Glossy15,

456 which was highly expressed in floating and submerged leaves, suggesting a similar function of

457 ERF in leaves of P. octandrus and maize.

458 The KNOX homeobox protein (Knotted1-like homebox, KNOX) is a homeodomain

459 transcription factor that maintains cell pluripotency in plant shoot apical meristems (SAM)

460 (Vollbrecht et al., 1991). Three KNOTTED1-like HOMEOBOX (KNOX) homologs were detected

461 in our DEG analysis, and all of these were highly expressed in leaf shoots. KNOX proteins

462 regulate the homeostasis of CTK and GA in order to maintain meristematic cells in an

463 undifferentiated state (Shani, Yanai \& Ori, 2006). CTK is a plant hormone involved in cell

464 proliferation while GA controls leaf morphogenesis (Hooley, 1994; Mok \& Mok, 2001). These

465 results imply that regulation of GA levels by $K N O X 1$ genes is involved in regulating

466 heterophylly in P. octandrus.

467 bZIP TFs regulate a variety of plant development and abiotic resistance processes. For

468 example, AtbZIP1 from Arabidopsis regulates ABA signal transduction by binding ABA-

469 responsive elements (ABREs) and altering the expression of ABA-responsive genes (Sun et al.,

470 2011). Here, three ABRE binding factors (AREB/ABF) were identified that were differentially

471 expressed between leaf shoots, floating leaves and submerged leaves. These findings suggest that

472 morphological differences between heterophyllous leaves may be directed by genes from

473 multiple functional groups, such as bZIP genes. 
We also identified several other TFs involved in ABA signalling. For example, the NAC TF,

475

476

477

478

479

480

481

482

483

484

485

ANAC072, was detected and responds to exogenous ABA and may regulate ABA-responsive genes (Tran et al., 2004). Moreover, ABA regulates gene expression through additional TFs such as $M Y B, H D-Z F, B 3$ and $b H L H$ (Fujita et al., 2011). Our data indicate that diverse TFs may be involved in heterophyllous leaf development and that the genes are both down-regulated and upregulated, suggesting that TFs may be involved in different processes during heterophyllous leaf development.

\section{Conclusions}

Here, we describe the production, assembly, and annotation of transcriptomes from submerged and floating leaves of a heterophyllous aquatic plant, P. octandrus. We identified several genes that showed differential expression at different time points of development during the onset of heterophylly in this species. These results inform on a more complete understanding of the molecular mechanisms underlying heterophylly in plants. In particular, these results can be used for cloning and functional studies of genes that are involved in heterophylly development in order to further probe the mechanistic basis for this important developmental phenomenon.

\section{REFERENCES}

Aharoni A, Dixit S, Jetter R, Thoenes E, van Arkel G, Pereira A. 2004. The SHINE clade of AP2 domain transcription factors activates wax biosynthesis, alters cuticle properties, and confers drought tolerance when overexpressed in Arabidopsis. Plant Cell 16: 2463-2480.

Allsopp A. 1962. The effects of gibberellic acid on morphogenesis in Marsilea drummondii. Phytomorphology 12: 1-10. 
495 Anderson LW. 1978. Abscisic acid induces formation of floating leaves in the heterophyllous 496 aquatic angiosperm Potamogeton nodosus. Science 201: 1135-1138.

497 Arber A. 1920. Water Plants: A Study of Aquatic Angiosperms. Cambridge University Press.

498 Audic S, Claverie JM. 1997. The significance of digital gene expression profiles. Genome $499 \quad$ Research 7: 986-995.

500 Banno H, Ikeda Y, Niu QW, Chua NH. 2001. Overexpression of Arabidopsis ESR1 induces 501 initiation of shoot regeneration. Plant Cell 13: 2609-2618.

502 Bodkin PC, Spence D, Weeks DC. 1980. Photoreversible control of heterophylly in Hippuris $503 \quad$ vulgaris L. New Phytologist 84: 533.

504 Chen H, Hwang S, Chen S, Shii C, Cheng W. 2011. ABA-mediated heterophylly is regulated by 505 differential expression of 9-cis-epoxycarotenoid dioxygenase 3 in lilies. Plant and Cell $506 \quad$ Physiology 52: 1806-1821.

507 Conesa A, Götz S, García-Gómez JM, Terol J, Talón M, Robles M. 2005. Blast2GO: a universal 508 tool for annotation, visualization and analysis in functional genomics research. 509 Bioinformatics 21: 3674-3676.

510 Cook SA, Johnson MP. 1968. Adaptation to heterogenous environments I. Variation in 511 heterophylly in Ranunculus flammula L. Evolution 22: 496-516.

512 Cutler SR, Rodriguez PL, Finkelstein RR, Abrams SR. 2010. Abscisic acid: emergence of a core 513 signaling network. Annual Review of Plant Biology 61: 651-679.

514 Deschamp PA, Cooke TJ. 1984. Causal mechanisms of leaf dimorphism in the aquatic 515 angiosperm Callitriche heterophylla. American Journal of Botany 71: 319-329.

516 Deschamp PA, Cooke TJ. 1985. Leaf dimorphism in the aquatic angiosperm Callitriche 517 heterophylla. American Journal of Botany 72: 1377-1387. 
518 Fassett NC. 1930. A Manual of Aquatic Plants. The University of Wisconsin Press.

519 Fujita Y, Fujita M, Shinozaki K, Yamaguchi-Shinozaki K. 2011. ABA-mediated transcriptional

520 regulation in response to osmotic stress in plants. Journal of Plant Research 124: 509-525.

521 Gee D, Anderson L. 1996. ABA induced differences during leaf development in the aquatic 522 angiosperm, Potamogeton nodosus, are detected with differential display. Plant Physiology 111: 110 .

524 Geiger D, Maierhofer T, Al-Rasheid KA, Scherzer S, Mumm P, Liese A, Ache P, Wellmann C,

525 Marten I, Grill E, Romeis T, Hedrich R. 2011. Stomatal closure by fast abscisic acid

526 signaling is mediated by the guard cell anion channel SLAH3 and the receptor RCAR1.

$527 \quad$ Science Signaling 4 (173): ra32.

528 Goliber T, Feldman L. 1989. Osmotic stress, endogenous abscisic acid and the control of leaf 529 morphology in Hippuris vulgaris L. Plant Cell and Environment 12: 163-171.

530 Grabherr MG, Haas BJ, Yassour M, Levin JZ, Thompson DA, Amit I, Adiconis X, Fan L, 531 Raychowdhury R, Zeng Q, Chen Z, Mauceli E, Hacohen N, Gnirke A, Rhind N, di Palma F, 532 Birren BW, Nusbaum C, Lindblad-Toh K, Friedman N, Regev A. 2011. Full-length 533 transcriptome assembly from RNA-Seq data without a reference genome. Nature 534 Biotechnology 29: 130-644.

535 Guo YH., Haynes RR., Hellquist CB., Kaplan Z. 2010. Potamogetonaceae. In: Wu ZY, Peter 536 HR. (Eds.) Flora of China. Volume 23. Science Press and Missouri Botanical Garden Press, 537 Beijing and St. Louis, 91-102.

538 Hay A, Tsiantis M. 2006. The genetic basis for differences in leaf form between Arabidopsis 539 thaliana and its wild relative Cardamine hirsuta. Nature Genetics 38: 942-947. 
540 Hirayama T, Shinozaki K. 2010. Research on plant abiotic stress responses in the post-genome

$541 \quad$ era: past, present and future. The Plant Journal 61:1041-1052.

542 Hooley R. 1994. Gibberellins: perception, transduction and responses. Plant Molecular Biology

$543 \quad 26: 1529-1555$.

544 Hsu TC, Liu HC, Wang JS, Chen RW, Wang YC, Lin BL. 2001. Early genes responsive to 545 abscisic acid during heterophyllous induction in Marsilea quadrifolia. Plant Molecular $546 \quad$ Biology 47: 703-715.

547 Iuchi S, Kobayashi M, Taji T, Naramoto M, Seki M, Kato T, Tabata S, Kakubari Y, Yamaguchi-

548 Shinozaki K, Shinozaki K. 2001. Regulation of drought tolerance by gene manipulation of 9-

549 cis-epoxycarotenoid dioxygenase, a key enzyme in abscisic acid biosynthesis in Arabidopsis.

$550 \quad$ The Plant Journal 27: 325-333.

551 Jackson D, Veit B, Hake S. 1994. Expression of maize KNOTTED1 related homeobox genes in

552 the shoot apical meristem predicts patterns of morphogenesis in the vegetative shoot.

553 Development 120: 405-413.

554 Kane ME, Albert LS. 1987. Abscisic acid induces aerial leaf morphology and vasculature in

555 submerged Hippuris vulgaris L. Aquatic Botany 28: 81-88.

556 Kanehisa M, Goto S. 2000. KEGG: Kyoto encyclopedia of genes and genomes. Nucleic Acids

$557 \quad$ Research 28: 27-30.

558 Kuwabara A, Ikegami K, Koshiba T, Nagata T. 2003. Effects of ethylene and abscisic acid upon

559 heterophylly in Ludwigia arcuata (Onagraceae). Planta 217: 880-887.

560 Kuwabara A, Tsukaya H, Nagata T. 2001. Identification of factors that cause heterophylly in

561 Ludwigia arcuata Walt.(Onagraceae). Plant Biology 3: 98-105. 
562 Langmead B, Salzberg SL. 2012. Fast gapped-read alignment with Bowtie 2. Nature Methods 9:

$563 \quad 357-359$.

564 Lin BL, Yang WJ. 1999. Blue light and abscisic acid independently induce heterophyllous

565 switch in Marsilea quadrifolia. Plant Physiology 119: 429-434.

566 Liu BL. 1984. Abscisic acid induces land form characteristics in Marsilea quadrifolia L.

567 American Journal of Botany 71: 638-644.

568 Livak KJ, Schmittgen TD. 2001. Analysis of relative gene expression data using real-time

569 quantitative PCR and the 2- $\Delta \Delta \mathrm{Ct}$ method. Methods 25: 402-408.

570 Marioni JC, Mason CE, Mane SM, Stephens M, Gilad Y. 2008. RNA-seq: an assessment of

571 technical reproducibility and comparison with gene expression arrays. Genome Research 18 :

$572 \quad 1509-1517$.

573 McCallum WB. 1902. On the nature of the stimulus causing the change of form and structure in

574 Prosperpinaca palustris. Botanical Gazette 34:93-108.

575 Minorsky PV. 2003. Heterophylly in aquatic plants. Plant Physiology 133: 1671-1672.

576 Mok D, Mok MC. 2001. Cytokinin metabolism and action. Annual Review of Plant Physiology

577 and Plant Molecular Biology 52: 89-118.

578 Moon J, Hake S. 2011. How a leaf gets its shape. Curr. Opin. Plant Biol. 14: 24-30.

579 Moose SP, Sisco PH. 1996. Glossy15, an APETALA2-like gene from maize that regulates leaf

$580 \quad$ epidermal cell identity. Genes \& Development 10: 3018-3027.

581 Mutasa-Goettgen, ES, Joshi A, Holmes HF, Hedden P, Goettgens B. 2012. A new RNASeq-

582 based reference transcriptome for sugar beet and its application in transcriptome-scale

583 analysis of vernalization and gibberellin responses. BMC Genomics 13: 99. 
584 Nakayama H, Nakayama N, Nakamasu A, Sinha N, Kimura S. 2012. Toward elucidating the 585 mechanisms that regulate heterophylly. Plant Morphology. 24: 57-63.

586 Nakayama H, Nakayama N, Seiki S, Kojima M, Sakakibara H, Sinha N, Kimura S. 2014.

587 Regulation of the $K N O X-G A$ gene module induces heterophyllic alteration in North $588 \quad$ American Lake Cress. Plant Cell 26: 4733-4748.

589 Olsen J L, Rouzé P, Verhelst B, Lin YC, Bayer T, Collen J, Dattolo E, De Paoli E, Dittami S, 590 Maumus F, Michel G, Kersting A, Lauritano C, Lohaus R, Töpel M, Tonon T, Vanneste K, 591 Amirebrahimi M, Brakel J, Boström C, Chovatia M, Grimwood J, Jenkins JW, Jueterbock A, 592 Mraz A, Stam WT, Tice H, Bornberg-Bauer E, Green PJ, Pearson GA, Procaccini G, Duarte 593 CM, Schmutz J, Reusch TB, Van de Peer Y. 2016. The genome of the seagrass Zostera marina reveals angiosperm adaptation to the sea. Nature, 530: 331-335.

Ozsolak F, Milos PM. 2011. RNA sequencing: advances, challenges and opportunities. Nature Reviews Genetics 2: 87-98.

597 Pigliucci M. 2010. Phenotypic plasticity. In: Pigliucci M, Muller GM, editors. Evolution: the 598 extended synthesis. MIT Press; p. 355-378.

599 Sculthorpe CD. 1967. The Biology of Aquatic Vascular Plants. Arnold E.

600 Shani E, Ben-Gera H, Shleizer-Burko S, Burko Y, Weiss D, Ori N. 2010. Cytokinin regulates 601 compound leaf development in Tomato. Plant Cell 22: 3206-3217.

602 Shani E, Yanai O, Ori N. 2006. The role of hormones in shoot apical meristem function. Current 603 Opinion in Plant Biology 9: 484-489.

604 Shi C, Yang H, Wei CL, Yu O, Zhang ZZ, Jiang CJ, Sun J, Li YY, Chen Q, Xia T, Wan XC. 605 2011. Deep sequencing of the Camellia sinensis transcriptome revealed candidate genes for 606 major metabolic pathways of tea-specific compounds. BMC Genomics 12: 131. 
607 Smith LG, Greene B, Veit B, Hake S. 1992, A dominant mutation in the maize homeobox gene, 608 Knotted-1, causes its ectopic expression in leaf cells with altered fates. Development 116: 2160930.

610 Sun X L, Li Y, Cai H, Bai X, Ji W, Ji ZJ, Zhu YM. 2011. Arabidopsis bZIP1 transcription factor 611 binding to ABRE cis-element regulates abscisic acid signal transduction. Acta Agronomica 612 Sinica. 37: 612-619.

613 Titus JE, Sullivan PG. 2001. Heterophylly in the yellow waterlily, Nuphar variegata 614 (Nymphaeaceae): effects of $\left[\mathrm{CO}_{2}\right]$ natural sediment type, and water depth. American Journal 615 of Botany 88: 1469-1478.

616 Tran L, Nakashima K, Sakuma Y, Simpson SD, Fujita Y, Maruyama K, Fujita M, Seki M, 617 Shinozaki K, Yamaguchi-Shinozaki K. 2004. Isolation and functional analysis of 618 Arabidopsis stress-inducible NAC transcription factors that bind to a drought-responsive $c i s-$ 619 element in the early responsive to dehydration stress 1 promoter. Plant Cell. 16: 2481-2498. 620 Tsukaya H. Mechanism of leaf-shape determination. Annual Review of Plant Biology 2006; 57: $621 \quad 477-496$.

622 Uchida N, Kimura S, Koenig D, Sinha N. 2010. Coordination of leaf development via regulation 623 of KNOX1genes. Journal of Plant Research 123:7-14.

624 Uchida N, Townsley B, Chung KH, Sinha N. 2007. Regulation of SHOOT MERISTEMLESS

625 genes via an upstream-conserved noncoding sequence coordinates leaf development. 626 Proceedings of the National Academy of Sciences of America. 104: 15953-15958.

627 van der Graaff E, Den Dulk-Ras A, Hooykaas P, Keller B. 2000. Activation tagging of the 628 LEAFY PETIOLE gene affects leaf petiole development in Arabidopsis thaliana. 629 Development. 127: 4971-4980. 
630 Varshney R, Nayak S, May G, Jackson S. 2009. Next-generation sequencing technologies and

631 their implications for crop genetics and breeding. Trends in Biotechnology 27: 522-530.

632 Vollbrecht E, Veit B, Sinha N, Hake S. 1991. The developmental gene Knotted-1 is a member of 633 a maize homeobox gene family. Nature 350: 241-243.

634 Walton DC, Li Y. 2013. Abscisic acid biosynthesis and metabolism. In: Davies P, editor. Plant 635 hormones: Physiology, Biochemistry and Molecular Biology. Springer Science \& Business 636 Media; p.140-157.

637 Wanke D. 2011. The ABA-mediated switch between submersed and emersed life-styles in 638 aquatic macrophytes. Journal of Plant Research 124: 467-475.

639 Wells CL, Pigliucci M. 2000. Adaptive phenotypic plasticity: the case of heterophylly in aquatic 640 plants. Perspectives in Plant Ecology Evolution and Systematics 3: 1-18.

641 Winn AA. 1999. Is seasonal variation in leaf traits adaptive for the annual plant Dicerandra 642 linearifolia? Journal of Evolutionary Biology 1999; 12: 306-313.

643 Winn AA. The functional significance and fitness consequences of heterophylly. International $644 \quad$ Journal of Plant Sciences 160: S113-S121.

645 Yang M, Zhu L, Xu L, Pan C, Liu Y. 2014. Comparative transcriptomic analysis of the 646 regulation of flowering in temperate and tropical lotus (Nelumbo nucifera) by RNA-Seq. 647 Annals of Applied Biology 165: 73-95.

648 Ye J, Fang L, Zheng H, Zhang Y, Chen J, Zhang Z, Wang J, Li ST, Li RQ, Bolund L, Wang J. 649 2006. WEGO: a web tool for plotting GO annotations. Nucleic Acids Research 34: W293$650 \quad$ W297.

651 Young JP, Dengler NG, Horton RF. 1987. Heterophylly in Ranunculus flabellaris: The effect of 652 abscisic acid on leaf anatomy. Annals of Botany 60: 117-125. 
653 Young JP, Horton RF. 1985. Heterophylly in Ranunculus flabellaris: the effect of abscisic acid. 654 Annals of Botany 55: 899-902.

655 Yuan L, Perry SE. 2011. Plant transcription factors. Methods and Protocols. Methods in $656 \quad$ Molecular Biology 754: 347.

657 Zhang X, Gituru RW, Yang C, Guo YH. 2009. Variations of floral traits among different life 658 forms illustrate the evolution of pollination systems in Potamogeton species from China. 659 Aquatic Botany 90: 124-128.

660 Zotz G, Wilhelm K, Becker A. 2011. Heteroblasty-a review. Botanical Review 77: 109-151. 661 


\section{Figure legends:}

663 Fig. 1 Morphological features of P. octandrus. (A) The initial developmental stage of the plant

664 that produces only submerged leaves. (B) The later developmental stage of the plant that

665 produces both floating and submerged leaves.

666 Fig. 2 Gene ontology (GO) annotations of all detected genes. Three main categories including

667 "biological process", "cellular component", and "molecular function" were summarized.

668 Fig. 3 DEGs identified in comparisons among different $P$. octandrus leaf development stages.

669 “Т01, Т04, Т12”, “Т02, Т03, Т05”, “Т06, Т08, Т13”, “Т07, Т10, Т14”, and “Т08, Т11, Т15”

670 indicate three biological replicates of shoot, earlier development floating leaves, later

671 development floating leaves, earlier development submerged leaves, and later development

672 submerged leaves, respectively. (A) Venn diagram showing common and unique DEGs among

673 different comparisons of floating leaves. (B) Venn diagram showing common and unique DEGs

674 among different comparisons of submerged leaves. (C) Expression patterns of DEGs among

675 different comparisons. The numbers above each bar indicate the total number of genes in each

676 group.

677 Fig.4 GO functional classifications of DEGs identified from comparisons among different 678 groups during floating leaf development. "T01, T04, T12”, “T02, T03, T05”, and “T06, T08,

679 T13" indicate three biological replicates of shoot, earlier development floating leaves, and later 680 development floating leaves, respectively.

681 Fig. 5 GO functional classifications of DEGs identified from comparisons among different 682 groups during submerged leaf development. “T01, T04, T12”, “T07, T10, T14”, and “T08, T11, 683 T15" indicate three biological replicates of shoot, earlier development submerged leaves, and 684 later development submerged leaves, respectively. 
685 Fig. 6 Dendrogram showing similarities in transcription factor expression profiles among

686 samples. Three transcription factor cluster groups (G1, G2 and G3) resulted from the 469

687 significantly differentially expressed transcription factors in leaves from different points of leaf

688 development.

689 Fig. 7 Distribution of transcription factor families among the three transcription factor cluster

690 groups (G1, G2 and G3).

691 
Figure 1

Morphological features of P. octandrus.

(A) The initial developmental stage of the plant that produces only submerged leaves. (B)

The later developmental stage of the plant that produces both floating and submerged leaves.
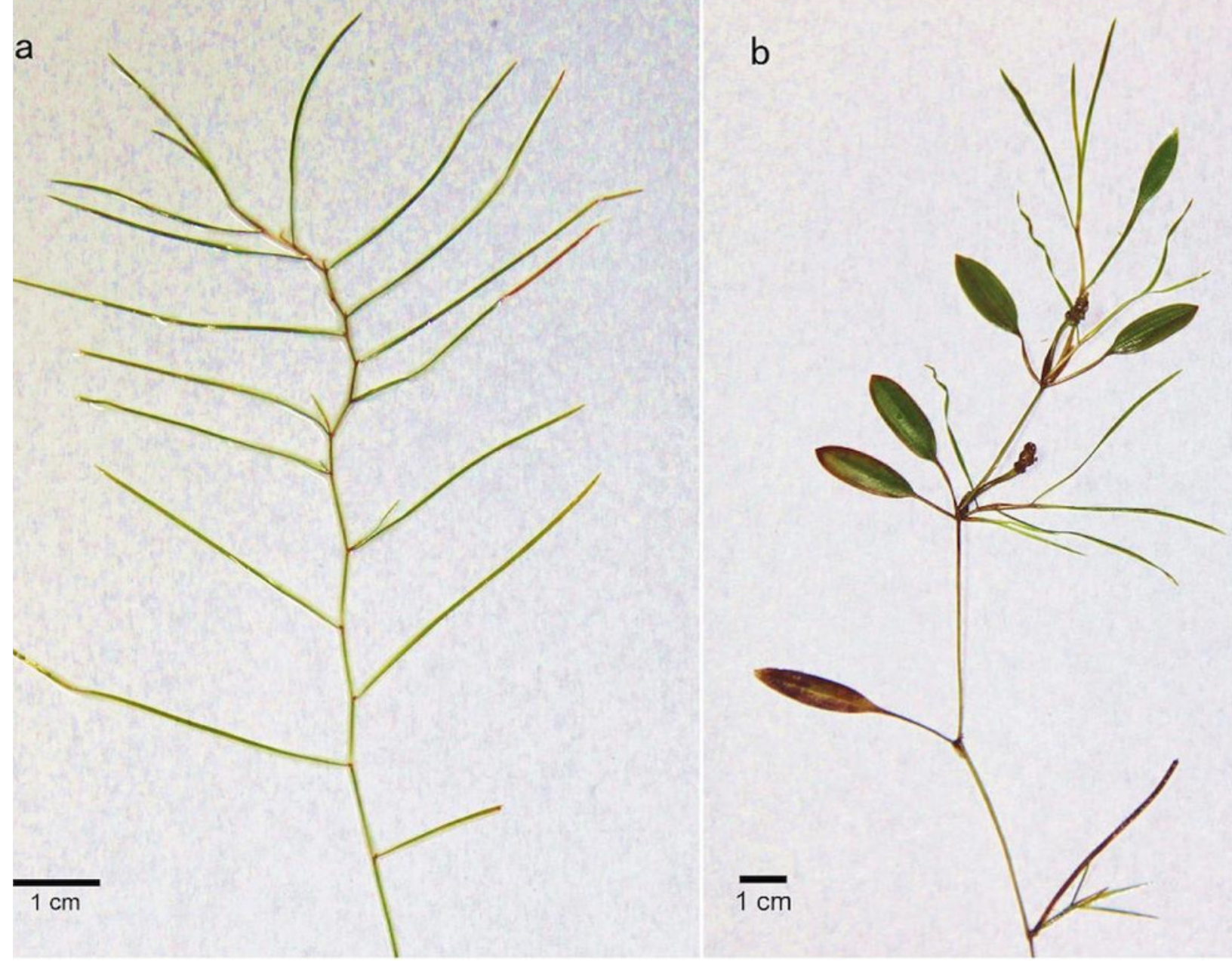
Figure 2

Gene ontology (GO) annotations of all detected genes.

Three main categories including "biological process", "cellular component", and "molecular function" were summarized.

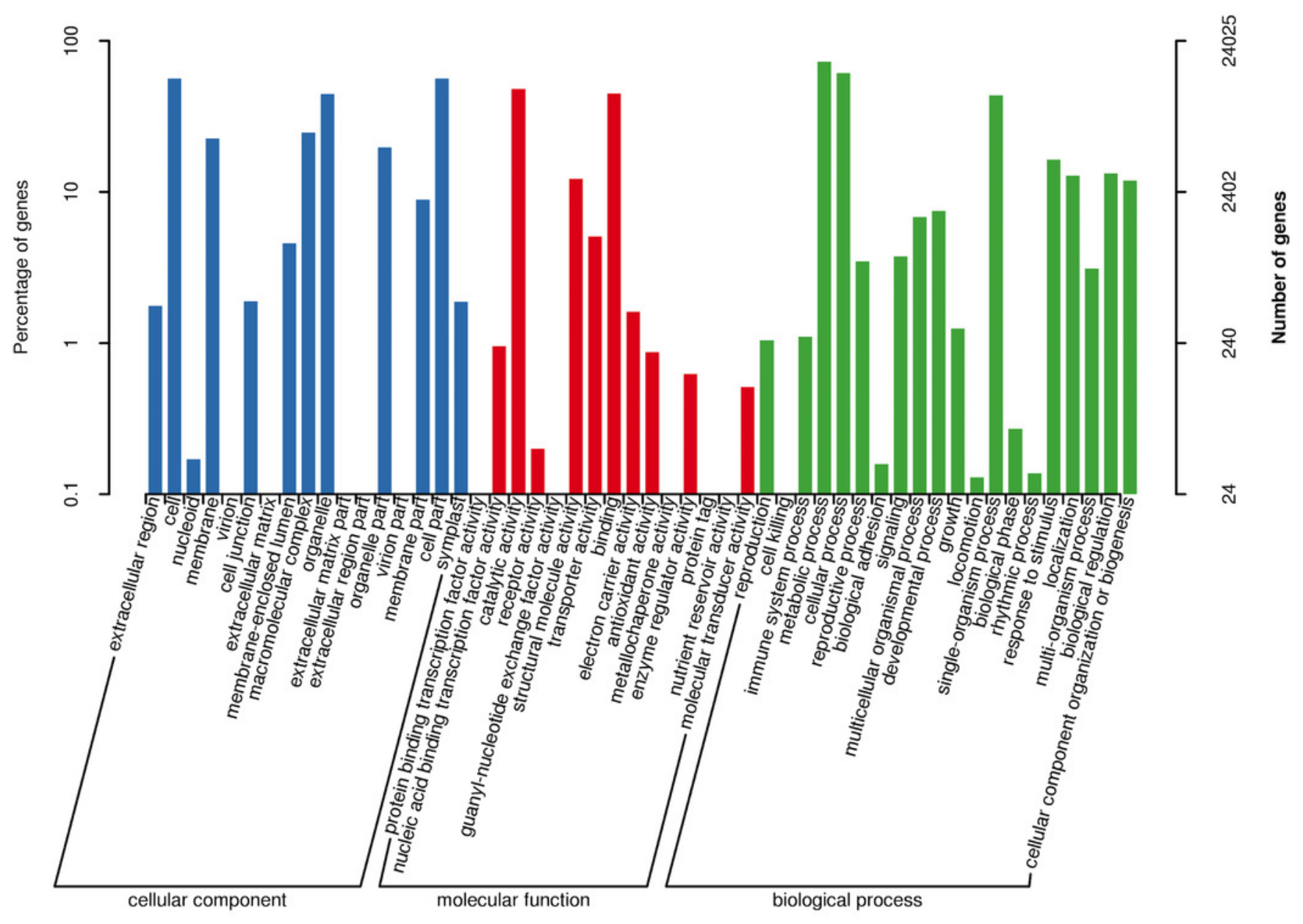




\section{Figure 3}

DEGs identified in comparisons among different P. octandrus leaf development stages.

“T01, T04, T12", “T02, T03, T05”, “T06, T08, T13", “T07, T10, T14”, and “T08, T11, T15” indicate three biological replicates of shoot, juvenile floating leaves, adult floating leaves, juvenile submerged leaves, and adult submerged leaves, respectively. (A) Venn diagram showing common and unique DEGs among different comparisons of floating leaves. (B) Venn diagram showing common and unique DEGs among different comparisons of submerged leaves. (C) Expression patterns of DEGs among different comparisons. The numbers above each bar indicate the total number of genes in each group. 

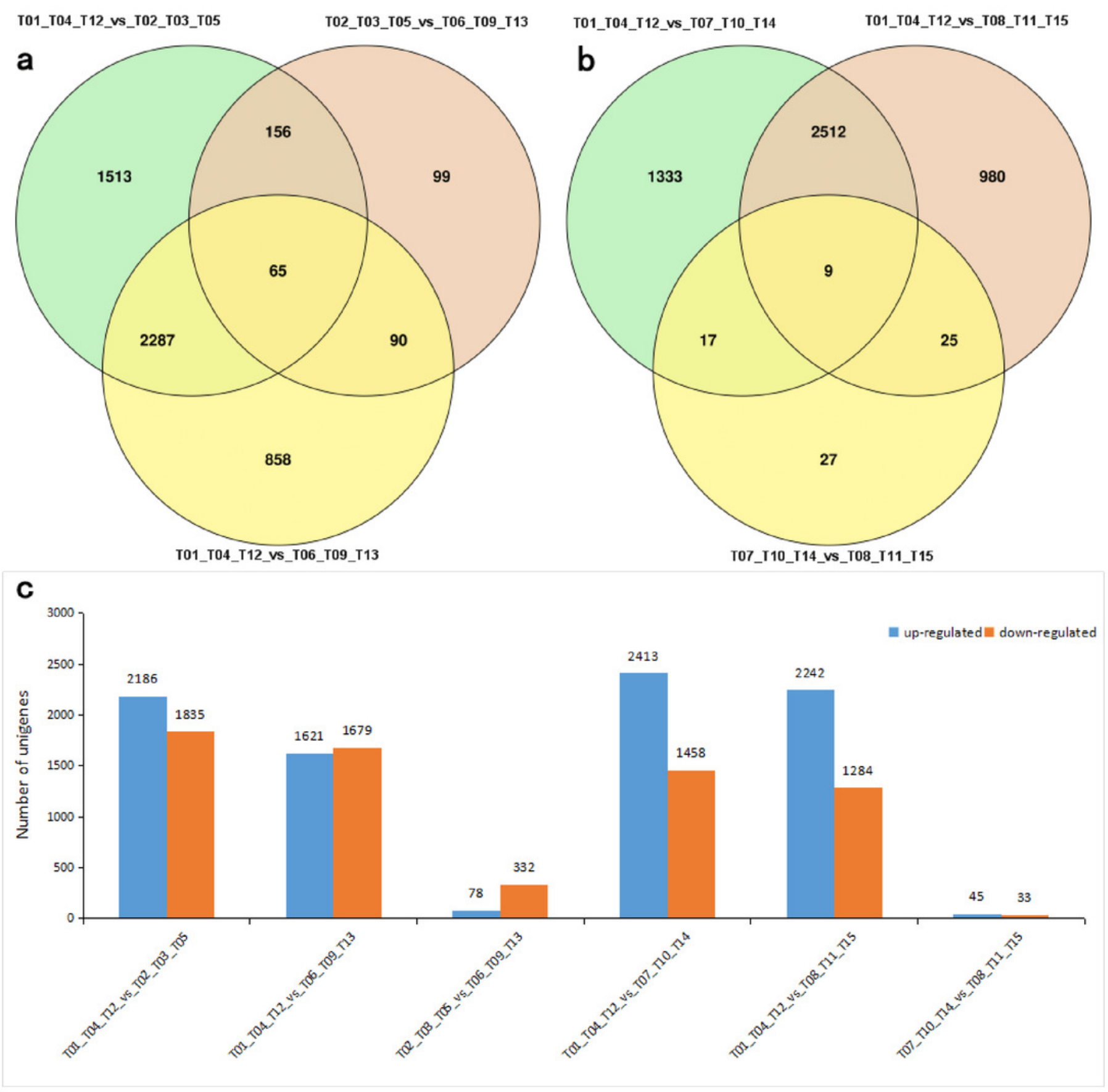
Figure 4

GO functional classifications of DEGs identified from comparisons among different groups during floating leaf development.

"T01, T04, T12", “T02, T03, T05", and "T06, T08, T13" indicate three biological replicates of shoot, juvenile floating leaves, and adult floating leaves, respectively.

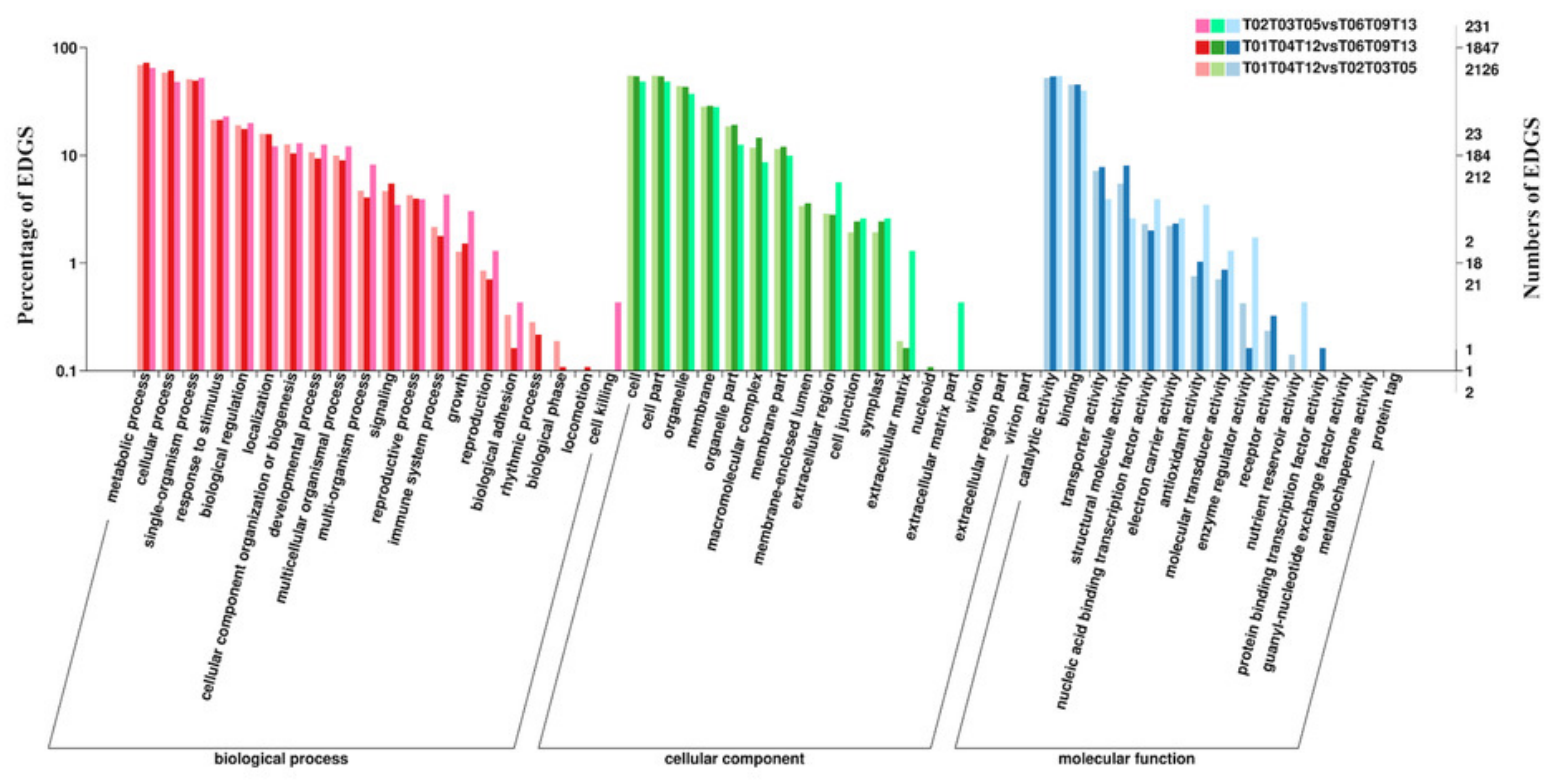


Figure 5

GO functional classifications of DEGs identified from comparisons among different groups during submerged leaf development.

"T01, T04, T12", "T07, T10, T14", and "T08, T11, T15" indicate three biological replicates of shoot, juvenile submerged leaves, and adult submerged leaves, respectively.

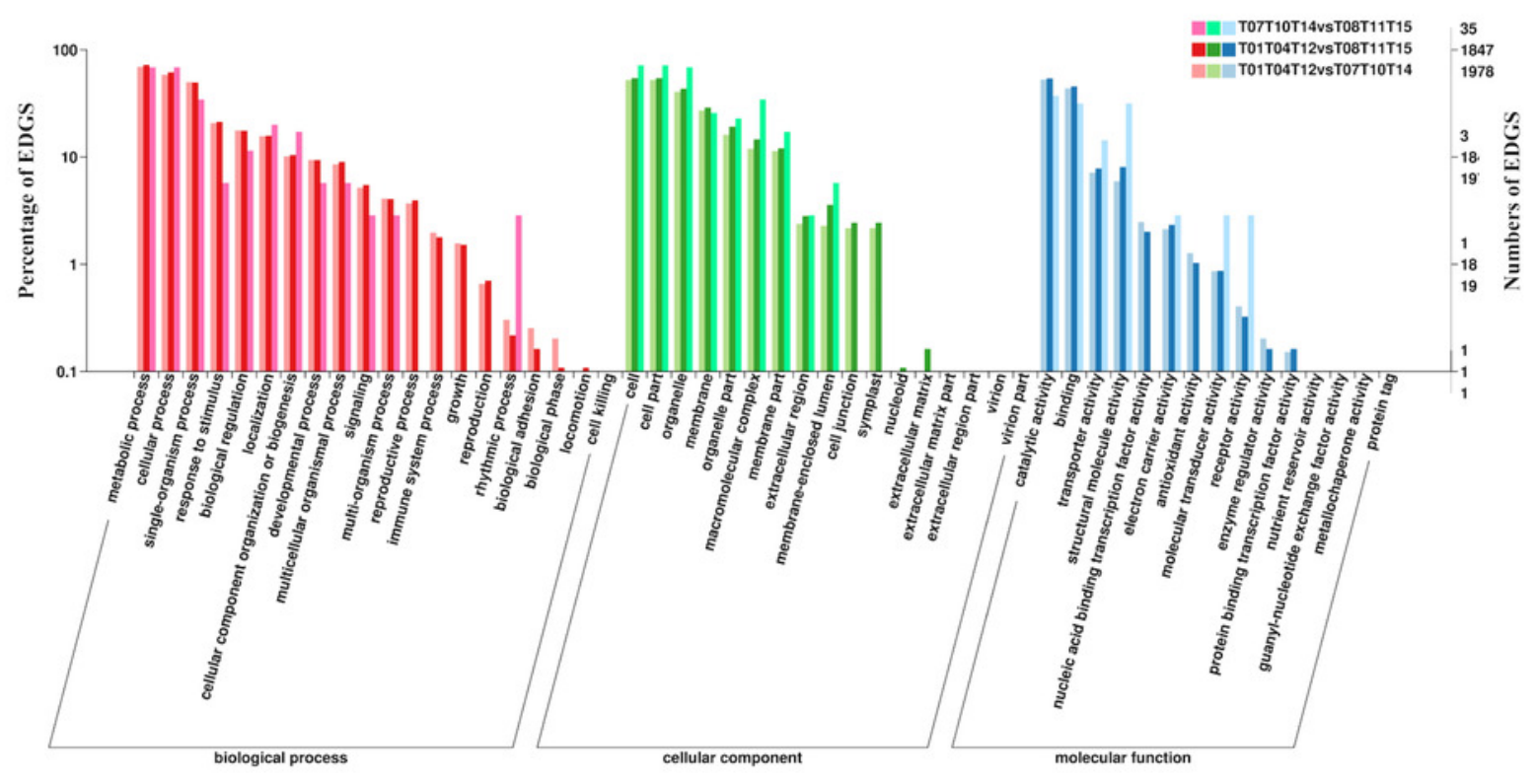




\section{Figure 6}

Dendrogram showing similarities in transcription factor expression profiles among samples.

Three transcription factor cluster groups (G1, G2 and G3) resulted from the 469 significantly differentially expressed transcription factors in leaves from different stages of development. 


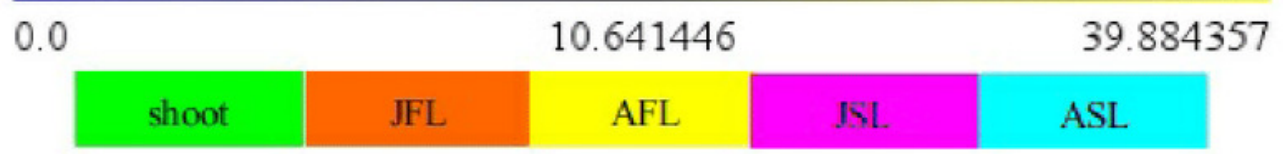

\section{T01 T04 T12 T02 T03 T05 T06 T09 T13 T07 T10 T14 T08 T11 T15}

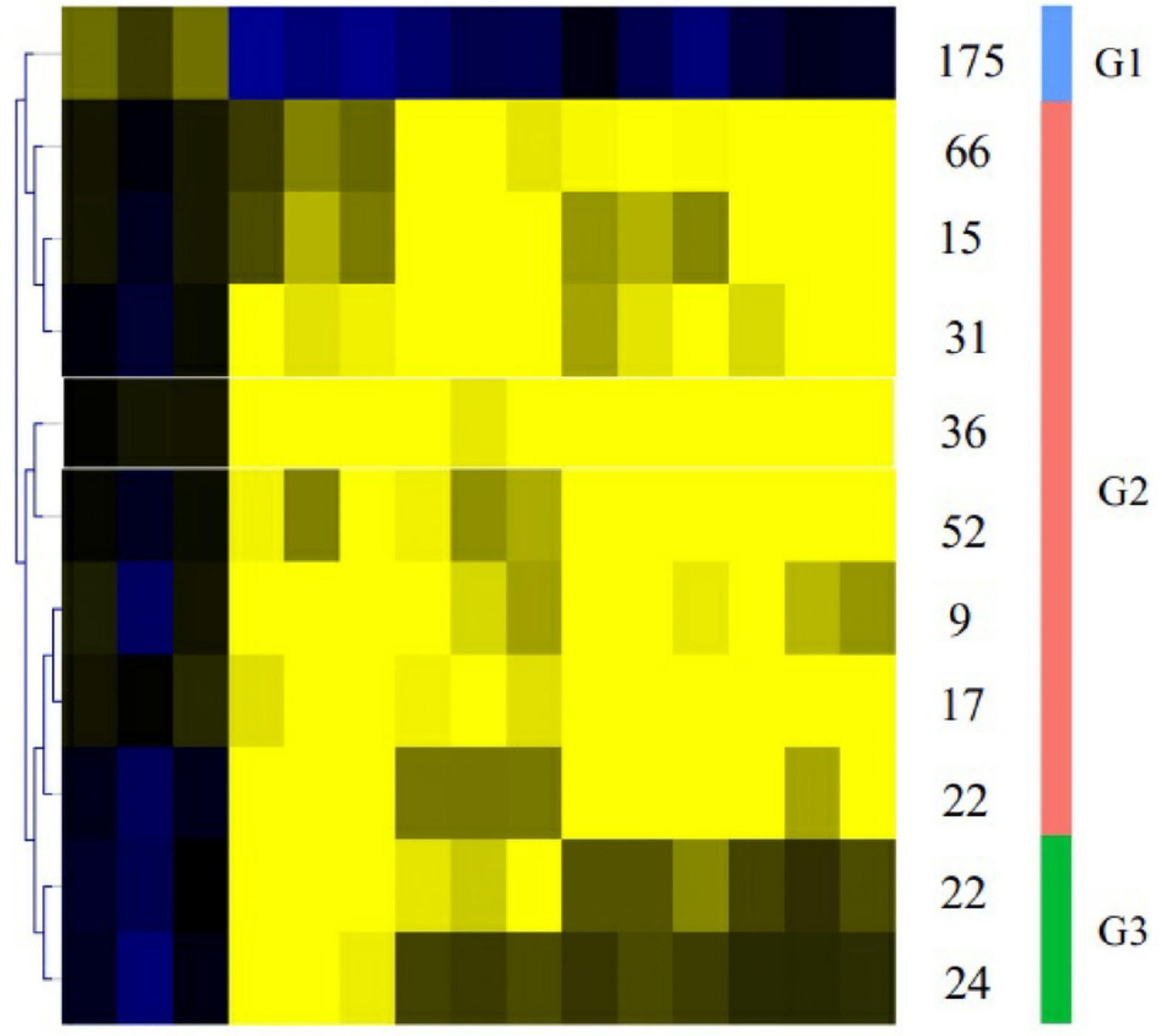


Figure 7

Distribution of transcription factor families among the three transcription factor cluster groups (G1, G2 and G3).

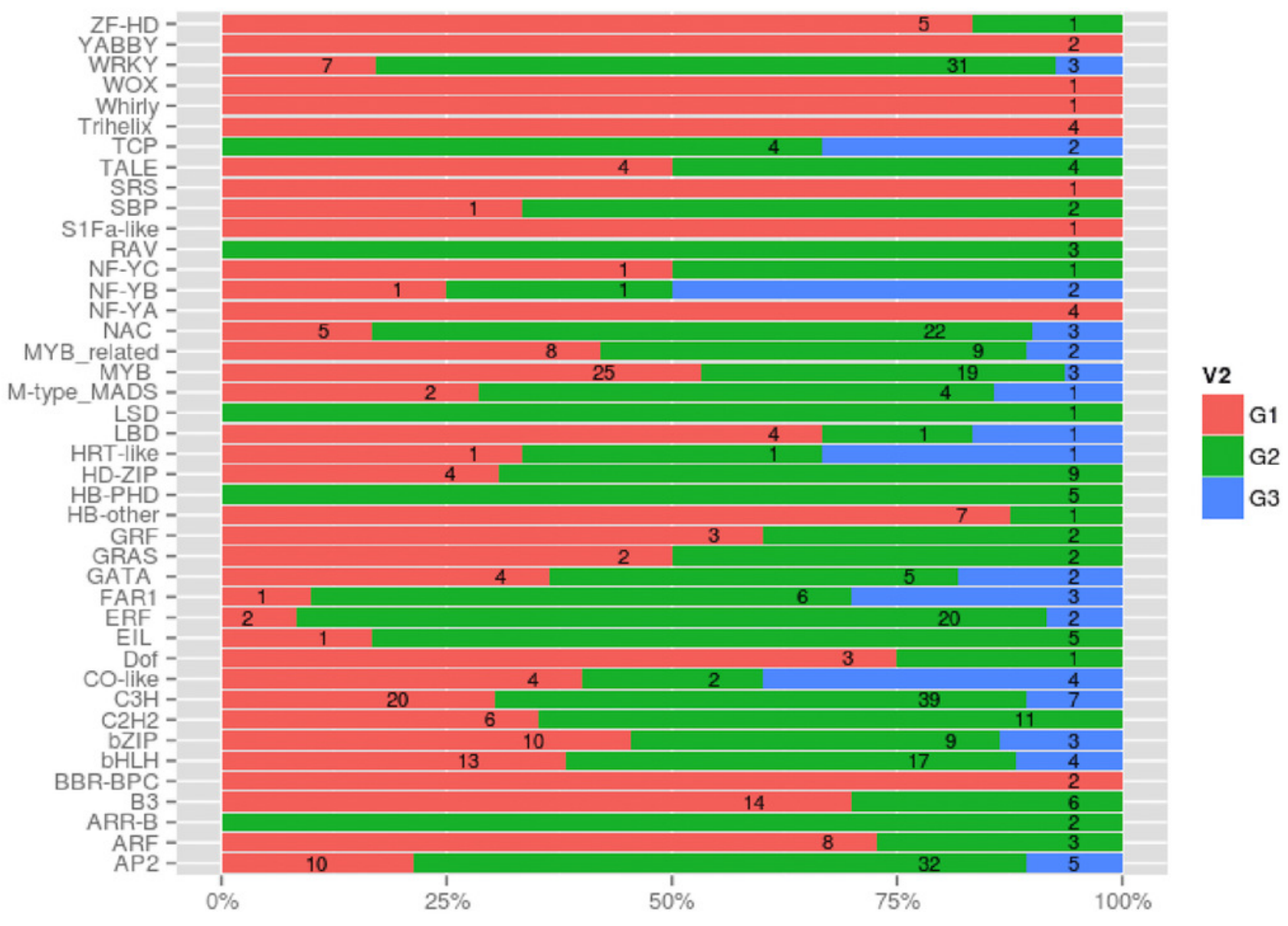




\section{Table 1 (on next page)}

Overview of the sequencing and assembly from RNA-Seq data. 
Table 1 Overview of the sequencing and assembly from RNA-Seq data.

\begin{tabular}{lll}
\hline Length & Transcript & Unigene \\
\hline $200-300$ & $40,102(23.97 \%)$ & $38,142(44.30 \%)$ \\
$300-500$ & $17,132(10.24 \%)$ & $11,774(13.67 \%)$ \\
$500-1000$ & $30,450(18.20 \%)$ & $15,041(17.47 \%)$ \\
$1000-2000$ & $39,384(23.55 \%)$ & $11,399(13.24 \%)$ \\
$2000+$ & $40,202(24.03 \%)$ & $9,747(11.32 \%)$ \\
Total Number & 167,270 & 86,103 \\
Total Length & $224,870,533$ & $72,422,038$ \\
N50 Length & 2,273 & 1,713 \\
Mean Length & 1344.36 & 841.11 \\
\hline
\end{tabular}

